\title{
Arterial oxygen saturation profiles in healthy preterm infants
}

A Ng, N Subhedar, R A Primhak, N J Shaw

\begin{abstract}
Aim-To construct a reference range of $\mathrm{SpO}_{2}$ values in healthy preterm infants using a simple data logging device.

Methods-Thirty three healthy preterm infants were monitored for a continuous period of 4 hours at rest using an Ohmeda Biox 3700 E Pulse Oximeter and an electronic data logger (Rustrack Ranger). Stored data were downloaded and saved as individual files on a personal computer. Results-The study group median and 5 th and 95th percentiles were used to construct a cumulative frequency curve of time against $\mathrm{SpO}_{2}$ value, representing the normal reference range of $\mathrm{SpO}_{2}$ profiles in healthy preterm infants.

Conclusion-Comparison of an infant's $\mathrm{SpO}_{2}$ profile against this curve may be more helpful in guiding supplemental oxygen treatment in that individual than a figure for a mean $\mathrm{SPO}_{2}$ and its standard deviation.

(Arch Dis Child Fetal Neonatal Ed 1998;79:F64-F66)
\end{abstract}

Keywords: oxygen saturation; data logging device

Infants with chronic lung disease (CLD) are prone to major complications such as acute life threatening events ${ }^{1}$ and pulmonary hypertension ${ }^{2} 3$ which may be related to chronic hypoxia.

In recent years monitoring of arterial oxygen saturation $\left(\mathrm{SpO}_{2}\right)$ using pulse oximetry has become the mainstay of assessment of oxygenation in preterm infants who have prolonged oxygen dependency. ${ }^{4}$ Although evaluation of the need for supplemental oxygen is invariably guided by measurements of $\mathrm{SpO}_{2}$, there is currently no agreement as to what level of $\mathrm{SpO}_{2}$ is acceptable in preterm infants with CLD. In the absence of any randomised trials comparing the safety and efficacy of different $\mathrm{SpO}_{2}$ levels maintained by supplemental oxygen therapy, one approach is to aim for normal $\mathrm{SpO}_{2}$ levels in these infants. ${ }^{67}$ There is, however, a lack of user friendly data on normal $\mathrm{SpO}_{2}$ in healthy preterm infants.

Previous studies attempting to define a normal reference range of $\mathrm{SpO}_{2}$ have used plethysmography and graphic analysis techniques. ${ }^{4}$ Although this methodology is undoubtedly meticulous, it is labour intensive and therefore impracticable for routine use in the neonatal nursery. Furthermore, data have been expressed as mean (SD) $\mathrm{SpO}_{2}$ rather than as a distribution of $\mathrm{SpO}_{2}$ values found with their relative frequencies of occurrence. A limitation of using most internal oximeter data storage modes is that they involve intermittent sampling or averaging techniques which may lead to data being erroneously lowered by movement artefact. We have recently studied the usefulness of a data logging device to assess $\mathrm{SpO}_{2}$ profiles. We describe our experience of using this system to collect data on $\mathrm{SpO}_{2}$ profiles, to construct a simple reference range of $\mathrm{SpO}_{2}$ values in healthy preterm infants.

\section{Methods}

Preterm infants who had received mechanical ventilation for less than six hours and supplemental oxygen for less than 48 hours were recruited after parental consent had been obtained. Infants with known congenital anomalies or cardiorespiratory disorders were excluded.

Demographic data were collected from maternal and infant case notes. Gestational age was assessed from maternal dates and/or early antenatal ultrasound examination. All infants were studied for a continuous period of four hours following a feed, while asleep, or during a period of quiet wakefulness. The infant was left undisturbed and the timing and duration of periods of activity (which may have led to signal artefact) were recorded separately by the nursing staff.

Arterial oxygen saturations and heart rate were continuously monitored using a Ohmeda Biox 3700e pulse oximeter with an averaging time of 3 seconds. The saturation probe was placed on the infant's hand or foot in a position that provided the most optimal signal.

An intelligent electronic datalogger (Rustrak Ranger, Outram Research, Bosham, UK) was connected to the analogue outputs for heart rate and saturation on the pulse oximeter. The logger was used in adaptive store mode, which has a sampling frequency of $2 \mathrm{~Hz}$, but a storage frequency that varies continuously according to the remaining memory and the variability of the signal. This storage mode permits the accurate recording of rapid changes in saturation or heart rate, and enables movement artefact to be distinguished.

Stored data were downloaded from the Rustrak Ranger and saved as individual files on a personal computer. Each infant's files of $\mathrm{SpO}_{2}$ and heart rate profiles were subsequently displayed in graphic form and analysed using dedicated software. After exclusion of obvious movement artefacts (defined as deflections to zero of both $\mathrm{SpO}_{2}$ and heart rate tracings), analyses were performed in a standardised manner by the same observer (AN); this analysis involved calculation of: (1) the total duration of the recording; (2) overall mean 


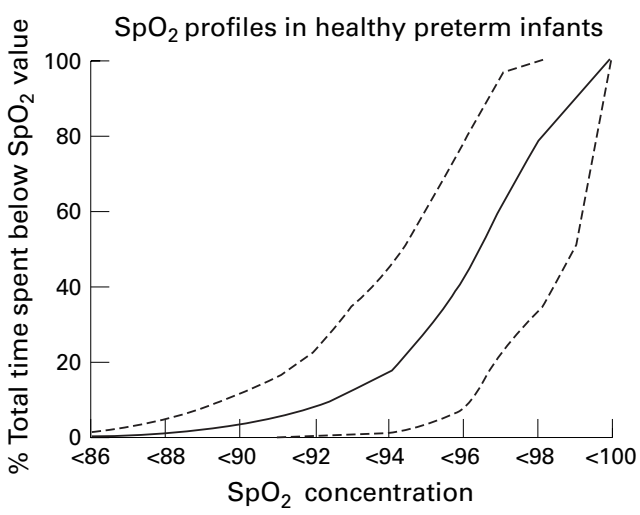

Figure 1

$\mathrm{SpO}_{2}$; and (3) cumulative time (expressed as a percentage of total time) spent below a range of individual $\mathrm{SpO}_{2}$ levels between 85 and $100 \%$. Using the study group median and 5 th and 95th percentiles, a cumulative frequency curve of time against $\mathrm{SpO}_{2}$ level was then constructed. This represented the normal reference range of $\mathrm{SpO}_{2}$ profiles in healthy preterm infants. Clinical staff were unaware of any of the results of the saturation studies.

Monitoring and analysis of $\mathrm{SpO}_{2}$ profiles was then performed in a similar way in a well preterm infant in whom it was no longer felt that there was a clinical need for oxygen and in an infant with CLD who was receiving supplementary oxygen. Individual $\mathrm{SpO}_{2}$ profiles were superimposed on the normal reference range to highlight any differences.

\section{Results}

Thirty three infants, 12 boys and 21 girls, were studied over a period of 7 months. Their median gestation was 33 (range 30-34) completed weeks and the median birthweight was 1798 (1020-2322) g. The median age at the time of study was 14 (range 2-28) days. No infant was receiving methylxanthines and all were breathing room air at the time of study. None had received a blood transfusion.

The median duration of monitoring (after exclusion of artefacts) was 240 (range 220 240) minutes. The median value of all the infants' mean $\mathrm{SpO}_{2}$ values was $97 \%$ (range $92-100 \%)$. Figure 1 shows the graph of cumulative time against $\mathrm{SpO}_{2}$ level for healthy preterm infants.

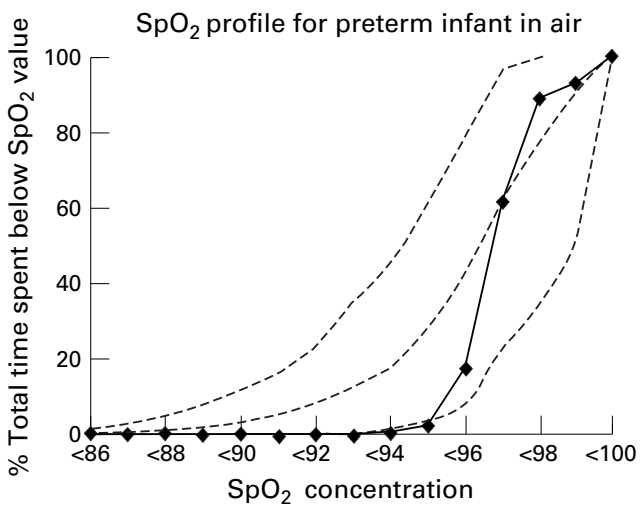

Figure 2

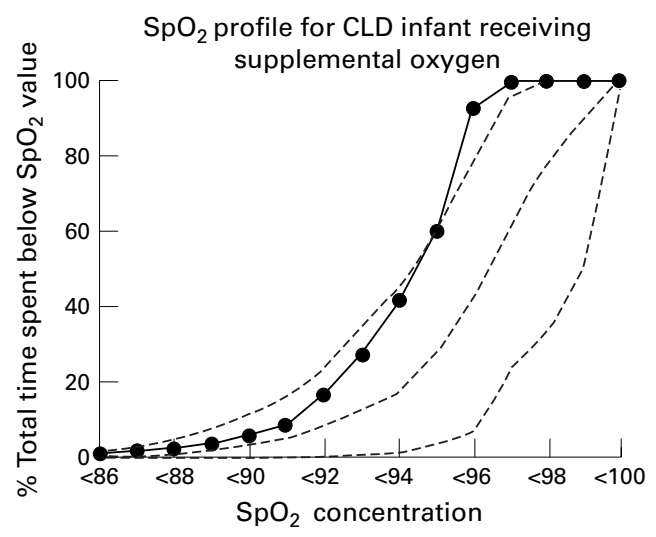

Figure 3

Examples of individual $\mathrm{SpO}_{2}$ profiles are shown in figs 2 and 3.

\section{Discussion}

In this study we have constructed a reference range of normal $\mathrm{SpO}_{2}$ distribution profiles in healthy preterm infants using a data logging device and dedicated software. The methodology used for analysing the data is relatively simple and practicable for routine use in the neonatal nursery.

The median of the mean $\mathrm{SpO}_{2}$ levels reported in this study (97\%) is lower than the baseline $\mathrm{SpO}_{2}$ value of $99.5 \%$ cited in a previous study in which a Nellcor N200 pulse oximeter was used. ${ }^{4}$ Calculated levels of $\mathrm{SpO}_{2}$ with the Ohmeda Biox III are about $2 \%$ lower than those obtained with the Nellcor N200, which may partly explain the discrepancy between the studies. ${ }^{8}$ If allowance is made for the different instruments used in the previous study, our results confirm that healthy preterm infants maintain relatively high baseline $\mathrm{SpO}_{2}$ values. The consistency of this finding is particularly surprising given the marked differences in pulse oximeter setup and analysis of $\mathrm{SpO}_{2}$ recordings in the two studies. Although we accept the desirability of detailed examinations of simultaneous $\mathrm{SpO}_{2}$, heart rate, and respiratory tracings, ${ }^{4}$ particularly to detect acute cardiorespiratory events, we feel that this methodology cannot routinely be used and is not relevant when the main objective is to detect persistent mild hypoxia.

None of our infants had received a blood transfusion before the study. It is likely, therefore, that all infants had predominantly haemoglobin $\mathrm{F}(\mathrm{HbF})$ in their blood. As $\mathrm{HbF}$ has a greater affinity for oxygen than haemoglobin A (HbA), it could be argued that normal $\mathrm{SpO}_{2}$ in our study infants could not be used to assess an older infant with predominantly $\mathrm{HbA}$ in their blood or one who had been transfused. However, previous studies in infants have suggested that the amount of $\mathrm{HbF}$ does not have a clinically significant effect on $\mathrm{SpO}_{2} \cdot{ }^{9}$

The cumulative frequency curve of oxygen saturations represents the range of $\mathrm{SpO}_{2}$ profiles in normal preterm infants. This curve may be more helpful in guiding supplemental oxygen treatment in infants with CLD as it provides a more visual user friendly display of 
the normal range of oxygen saturation than a single figure for a mean (SD) $\mathrm{SpO}_{2}$ does. For example, monitoring an infant in whom it is thought that supplemental oxygen treatment may be required in air over a period of a few hours would permit the construction of an individual $\mathrm{SpO}_{2}$ profile. By superimposing this individual profile on the group cumulative frequency curve, any deviation from the normal $\mathrm{SpO}_{2}$ profile pattern could be assessed. If the infant's profile shifts to the left (with a greater proportion of time spent at $\mathrm{SpO}_{2}$ levels below normal values), this might indicate a requirement for supplemental oxygen. The exact requirement could also be adjusted by repeating the process once the infant was receiving supplemental oxygen (as illustrated by the $\mathrm{SpO}_{2}$ curve of the infant in fig 3). Oxygen saturation profiles could also be produced for older children to enable infants with CLD to be assessed for their oxygen requirement after discharge from the neonatal unit. Further studies are required to assess whether the decision to stop an infant's supplementary oxygen, based on clinical examination and routine observations of oxygen saturations in the neonatal nursery, correlates with a normal oxygen saturation profile; and whether deviation from the oxygen saturation profile in infants with previous CLD who are breathing room air is associated with a poorer outcome.

Infants in this study were monitored for a continuous period of four hours while undisturbed. Recording of $\mathrm{SpO}_{2}$ levels during activities such as feeding and handling were not studied because of the potential inaccuracies related to movement artefact. Monitoring for a more prolonged period would inevitably have included such episodes, and would have required further detailed examination of $\mathrm{SpO}_{2}$ tracings to exclude artefacts. Furthermore, state of wakefulness and feeding in themselves may affect $\mathrm{SpO}_{2}$ values. ${ }^{70}$ Our aim in this study was to develop a method of assessing $\mathrm{SpO}_{2}$ profiles that was easy to use. Restricting the duration of recording to four hours permitted a reasonable period of study, while maintaining the practicability of the method. Although we excluded major movement artefact from the analysis, we cannot be certain that minor artefacts resulting in transient desaturation were detected. The most likely effect of including these events would have been to lower marginally the overall mean $\mathrm{SpO}_{2}$.

In summary, we have described a convenient, relatively inexpensive, and easy to use system to obtain $\mathrm{SpO}_{2}$ profiles in healthy preterm infants. Using this method we have constructed a normal reference range for $\mathrm{SpO}_{2}$. Comparison of an individual's $\mathrm{SpO}_{2}$ profile against these normal values may help to guide supplemental oxygen treatment in infants with CLD.

1 Iles R, Edmunds AT. Prediction of early outcome in resolving chronic lung disease of prematurity after discharge from hospital. Arch Dis Child 1996;74:304-8.

2 Gill AB, Weindling AM. Raised pulmonary artery pressure in very low birth weight infants requiring supplemental in very low birth weight infants requiring supplemental 1995;72:F20-F2

3 Benatar A, Clarke J, Silverman M. Pulmonary hypertension in infants with chronic lung disease: non-invasive evaluation and short-term effect of oxygen treatment. Arch Dis Child 1995;72:F14-F19.

4 Poets CF, Stebbens VA, Alexander JR, Arowsmith WA, Salfield SAW, Southall DP. Arterial oxygen saturation in preterm infants at discharge from the hospital and six weeks later. F Pediatr 1992;120:447-54.

5 Silva DT, Hagan R, Sly PD. Home oxygen management of neonatal chronic lung disease in Western Australia. 7 Paeneonatal chronic lung disease in
diatr Child Health 1995;31:185-8.

6 Hay WW, Thilo E, Curlander JB. Pulse oximetry in neonatal medicine. Clin Perinatol 1991;18:441-71.

7 Mok JYQ, McLaughlin FJ, Pintar M, Hak H, ArmaroGalvez R, Levison H. Transcutaneous monitoring of oxygenation: what is normal? f Pediatr 1986;108:365-71.

8 Poets CF, Southall DP. Non-invasive monitoring of oxygenation in infants and children: practical considerations and areas of concern. Pediatrics 1994;93:737-46.

9 Pologe JA, Raley DM. Effects of fetal haemoglobin on pulse oximetry. F Perinatol 1987;7:324-6.

10 Mok JYQ, Hak H, McLaughlin FJ, Pintar M, Canny GJ, Levison $H$. Effect of age and state of wakefulness on transcutaneous oxygen values in preterm infants: a longitudinal study. F Pediatr 1988;113:706-9. 